\title{
Does emotion before a chemotherapy course break a heart? Cardiogenic shock complicating Takotsubo cardiomyopathy
}

Karima Taamallah ( $\square$ karima.taamallah@fmt.utm.tn )

Military hospital of Tunis https://orcid.org/0000-0002-9904-2897

Hela Ghedira

Military hospital of Tunis

\section{Younes Arous}

Military hospital of Tunis

Abdeddayem Haggui

Military hospital of Tunis

Sarra Chenik

Military hospital of Tunis

Nadhem Hajlaoui

Military hospital of Tunis

Wafa Fehri

Military hospital of Tunis

\section{Case report}

Keywords: Takotsubo cardiomyopathy, chemotherapy, cancer, echocardiography, stress

Posted Date: January 13th, 2021

DOl: https://doi.org/10.21203/rs.3.rs-142578/v1

License: (9) This work is licensed under a Creative Commons Attribution 4.0 International License. Read Full License 


\section{Abstract}

Introduction: Takotsubo cardiomyopathy is characterized by a broad spectrum of clinical presentations. Despite its reversibility, it is associated with severe adverse in-hospital events. Recent studies have identified a high incidence of TTC in cancer patients.

Case report: We report the case of a 59-year-old-man with TTC occurring just before injecting of chemotherapy drug complicated by cardiogenic shock and acute heart failure. Under medical treatment, a complete recovery of cardiac contractility was noted within three days. chemotherapy regimen has been stopped and then alleviated from RCHOP to the RCVD. No recurrence of cardiac symptoms was noted during the follow-up period. Left ventricle ejection fraction, BNP, and troponin levels were maintained normal.

Conclusion: Takotsubo cardiomyopathy can occur in cancer patients leading to interruption of chemotherapy because of left ventricular dysfunction, which may adversely affect the oncologic outcome because of interruption of cancer treatment. We report this case to remind clinicians that TTC should be considered in the differential diagnosis of chest pain in cancer patients who present with ECG findings characteristic of the acute coronary syndrome. We hope to facilitate prompt diagnosis for an early intervention that allows rapid recovery of myocardial function, and resume cancer therapy early and may help lower mortality.

\section{Background}

Takotsubo cardiomyopathy (TTC), also known as stress-induced cardiomyopathy, is characterized by a broad spectrum of possible clinical presentations (1). Despite its reversibility, it is associated with severe adverse in-hospital events, the acute stage of the disease can be complicated by hypotension, shock, arrhythmias, heart failure, and death (2). Recent studies have identified a high incidence of TTC in cancer patients (3). TTS has been linked to emotional stress due to excessive release of catecholamines as a mechanism of response (1). The emotional stress of a cancer diagnosis, physical stress, ongoing chemotherapy, the abnormal cancer-related wasting syndrome may increase the risk of developing TTC. There have been very few, and small studies were addressing the prevalence and outcomes of cancer patients with TTC (4). We report the case of a patient with TTC occurring just before injecting of chemotherapy drug complicated by cardiogenic shock and acute heart failure. Given the extraordinary rarity of this condition, we report this case to remind the clinician to consider this condition in the differential diagnosis of acute coronary syndrome or cardiogenic shock in a cancer patient. We hope to facilitate an earlier diagnosis of TTC for a prompt treatment that allows the continuation of chemotherapy.

\section{Case Report}


A 59-year-old man was admitted to the department of hemato-oncology for chemotherapy for follicular non-Hodgkin's lymphoma diagnosed in May 2020, and had completed two cycles of chemotherapy of CHOP plus Rituximab regimen (doxorubicin, vincristine, cyclophosphamide, rituximab, and methylprednisolone), the cumulated dose of anthracycline was $100 \mathrm{mg} / \mathrm{m}^{2}$. The patient also had a history of diabetes mellitus and systemic arterial hypertension. He did not have any previous cardiac history; cardiac investigations done before starting chemotherapy were normal. Before the infusion of the third course of chemotherapy, the patient was stressed, he had pain because of a laborious arterial punction, he developed acute shortness of breath, discomfort, chest pain, and excessive sweating. He subsequently became tachycardic with a heart rate of $120 \mathrm{bpm}$, with rapid progression to cardiogenic shock requiring inotropic agents with blood pressure at $60 / 40 \mathrm{~mm} \mathrm{Hg}$. The patient was then transferred to an intensive care unit.

A 12-lead ECG showed a sinus tachycardia of $130 \mathrm{bpm}$, associated with ST-segment elevation in leads V1-V6. There was a rise in the level of serum troponin-I at $100 \mathrm{ng} / \mathrm{L}$ (normal range $0-40$ ). An urgent transthoracic bedside echocardiogram revealed normal-sized but apical ballooning with hyperdynamic basal segments. The estimated left ventricular (LV) ejection fraction (EF) was 38\%. There were no signs of tamponade and pulmonary embolism (figure 1). 2D strain was decreased at $-12.6 \%$ (figure 1). A provisional diagnosis of anterior ST-elevation myocardial infarction was made, coronary angiography was performed, demonstrated non-obstructive right coronary artery atheroma which did not explain the cardiogenic shock (figure 2). A subsequent cardiac MRI (figure 3) reported an akinetic left ventricle from the mid ventricle to the apex with impaired global ejection fraction, there were signs of myocardial edema in the LV walls in T2-weighted sequences, suggestive of Takotsubo cardiomyopathy, there was no evidence of myocardial inflammation, myocardial infarction, fibrosis, or infiltration in the late gadolinium enhancement sequences. Based on these findings, a diagnosis of TTC was made.

Treatment with $\beta$-adrenoceptor antagonists : Bisoprolol $1.25 \mathrm{mg}$ daily and ACE inhibitors: ramipril $2.5 \mathrm{mg}$ daily (titrating the doses up to the maximum doses tolerated by the patient) was initiated.

A remarkable improvement in LV function was noted in the echocardiogram performed three days after the event, LVEF and 2D strain became in the normal range (figure 1).

The patient remains in follow-up in the onco-cardiology unit, he was examined weekly, for four weeks after the event, and reported no recurrence of his symptoms. The chemotherapy regimen has been alleviated from RCHOP to the RCVD regimen which does not contain doxorubicin, known to be highly cardiotoxic; it was considered that its reintroduction in a patient with impaired myocardial function could be deleterious. After each course of chemotherapy, troponin and brain natriuretic peptide (BNP) remained at normal levels, and the size and function of the left ventricle were maintained normal.

\section{Discussion}


This report illustrates the case of a patient with lymphoma, treated with chemotherapy and presented TTS followed by cardiogenic shock during the preparation of the third course of chemotherapy.

Takotsubo cardiomyopathy, first described in the 1990s in Japan, is a form of reversible and transitory ventricular systolic dysfunction with a clinical and electrocardiographic presentation similar to acute coronary syndrome (ACS) or an acute heart failure (3). In general population, TTC is considered relatively uncommon with an incidence rate of $1 \%$ to $2 \%$, this incidence is higher in cancer patients (approximately at $10 \%$ ). Sattler et al. (5) reported that the prevalence of malignancy was high amongst TTC patients and that cancer was a risk factor for worse outcomes. One of the hypothesis is that cancer and TTS share similar triggering mechanisms, which consist in activation of the sympathetic nervous system (4).

This disease predominantly affects postmenopausal women (6), a similar incidence in men and women was noted in patients with TTC induced by cancer drugs (7). Giza et al. (3) reported that the most common cancers associated with TTC were lymphoproliferative neoplasms $(23.3 \%)$ which is the case of our patient, followed by gastrointestinal (17\%), ovarian (13.3\%), and breast cancer (10\%). The exact trigger for TTC is often challenging to identify. An emotional, physical, or combined trigger can precede the Takotsubo syndrome event, but this is not obligatory. The triggers which precipitate TTC in cancer patients are unknown, but a variety of factors have been described: chemotherapy, radiotherapy, radiofrequency ablation, and cancer pain (4). Although our patient outwardly displayed little anxiety about his diagnosis and treatment approach, it is possible that he felt a heightened degree of internal emotional stress that may have contributed to the development of TTC. The pain during the arterial punction could have triggered a cardiomyopathy induced by the release of several inflammatory cytokines and metabolites related to this physical distress.

Endothelial dysfunction in microvascular and epicardial coronary arteries might be a predisposing factor for the development of TTC, it occurs frequently in patients with cancer, especially during and after systemic chemotherapy or radiotherapy of the thoracic region, our patient had hypertension and diabetes mellitus and might had already endothelial dysfunction which might be worsened under chemotherapy (7).

Takotsubo cardiomyopathy remains a diagnostic challenge in cancer patients due to the difficulty in distinguishing TTC from chemotherapy-induced cardiomyopathy, as the two diseases differ in their management, reversibility, and long-term outcomes. Identifying reversible cardiomyopathy such as TTC in cancer patients is imperative, especially in patients who are undergoing curative cancer treatment. Indeed, recognition of chemotherapy-induced cardiotoxicity is important since repeated administration of the offending drug can potentially lead to irreversible cardiac damage. On the other hand, premature discontinuation of an effective anti-neoplastic agent due to co-existing cardiac events not directly related to therapy may increase oncologic morbidity and mortality. For these reasons, early diagnosis and appropriate management of TTC in patients with cancer are crucial. The classic wall motion abnormalities of the LV apical and mid segments which extend beyond a single coronary distribution or the typical appearance of "apical ballooning" provide a clue to the diagnosis of TTC. When LV contractile 
dysfunction is diffuse or global, it is challenging to distinguish Takotsubo syndrome from another type of chemotherapy-related cardiac dysfunction (8).

Certain chemotherapeutic agents have been implicated in triggering this form of cardiomyopathy. 5fluorouracil is the most commonly reported anti-neoplastic agents associated with TTC $(3,7)$. Other chemotherapeutic agents were also implicated such as Bevacizumab, Capecitabine, Bevacizumab, Combretastatin, Tyrosine kinase inhibitors, Miscellaneous drugs, Immune checkpoint inhibitors, Daunorubicin, Cytarabine, Trastuzumab, Ipilimumab, and Rituximab that our patient is taking as part of the $\mathrm{R}$ CHOP regimen (7). Previous usage of Rituximab might have contributed to the development of myocardial dysfunction in our patient. Two postulated mechanisms explaining the pathophysiology of rituximab-induced TTC have been proposed: Coronary vasospasm and direct cardiotoxic effect (9), and the presence of diffuse reticulin fibers along in cardiac myocytes after treatment with rituximab with increased levels of transforming growth factors (TGF) $-\beta$, explaining cardiac events during later infusions of rituximab (10).

TTC related to Vincristine, Doxorubicin, Cyclophosphamide (administered in our patient) was not reported. In the case of TTC in cancer patients under chemotherapy, the question of whether the cancer drug is the offender, a mere bystander, or paved the way by impairment of the myocardial function, is a crucial question that needs to be examined in a case-by-case scenario.

Rapid recovery of left ventricular function was observed in our patient, demonstrating the reversibility of this disease and the positive response to treatment. Recovery of systolic function typically occurs within one to four weeks (11). In patients with TTC occurring under chemotherapy, the recovery of a normal left ventricular function was noted within a mean duration of one month (range five days to three months) (7).

Our patient was maintained under $\beta$-adrenoceptor antagonists and ACE inhibitors, whether medical treatment can be discontinued after normalization of LVEF remains unknown. However, some authors supported the value of indefinitely continuing medical therapy by conducting a randomized trial, which assessed the effect of medical treatment withdrawal in patients who have recovered their LVEF after a dilated cardiomyopathy (12).

In a literature review conducted by Desai et al. (7) $44 \%$ of the patients in whom heart failure treatment was discontinued developed a relapse of cardiomyopathy during the first six months.

The chemotherapy regimen was alleviated in our patient. In patients who require further cancer treatment, rechallenging with the culprit anti-neoplastic therapy poses a significant clinical dilemma. There is limited data on the safety of reintroducing the chemotherapeutic agent. In a retrospective analysis of 30 patients with cancer and stress-induced cardiomyopathy treated at the MD Anderson Cancer Center (3), 21 patients required ongoing cancer treatment. Among them, 16 were able to safely resume chemotherapy after normalization of LVEF without recurrence of TTC. The median time to resume cancer treatment was 20 days after TTC. 
Given the worse outcomes in TTC patients with cancer and the difficulty in discriminating this cardiomyopathy from chemotherapy-induced cardiomyopathy and acute coronary syndrome, we believe that it is essential to develop an algorithm that includes imaging tests such as an echocardiogram, coronagraphy, MRI, to help make a timely diagnosis. The report of this case is an opportunity to stress the importance of pain control and emotional support in cancer patients.

\section{Conclusion}

In cancer patients, TTC should be considered in the differentialdiagnosis of chest pain, and electrocardiographic abnormalities without a culprit coronary artery lesion. Although the prognosis from TTC is generally good, this condition can lead to an interruption in chemotherapy, which may adversely affect the oncologic outcome. Early intervention may help lower mortality, rapid recovery of myocardial function, and resume cancer therapy early. Therefore, medical professionals need to recognize, monitor, and treat this condition appropriately.

\section{Declarations}

Conflict of interest: The authors declare that they have no potential conflicts of interest related to this publication.

No funding to declare.

\section{Authors' contributions:}

TAAMALLAH Karima, Ghdira Hela, AROUS Younes: Wrote the first draft of the manuscript.

TAAMALLAH Karima: Bibliography research

HAGGUI Abeddayem, HAJALOUI Nadhem, LAHIDHEB Dhaker: Contributed to the writing of the manuscript and bibliography research.

FEHRI Wafa, MSADDEK Fehmi: Made critical revisions and approved the final version.

All the authors reviewed and approved the final manuscript.

Availability of data and materials: Not applicable.

Ethics approval and consent to participate: This case report was approved by the ethics committee of the Military hospital, Tunis, Tunisia. Patient gave written consent to use his clinical and paraclinical information to prepare this article.

Consent for publication: The patient gave permission to publish her case, as well as relevant related workup and diagnostic images, presented in the medical literature. 
Acknowledgements: No acknowledgements to declare.

\section{References}

1. Pavin D, Le Breton H, Daubert C. Human stress cardiomyopathy mimicking acute myocardial syndrome. Heart Br Card Soc. 1997;78(5):509-11.

2. Singh K, Carson K, Shah R, Sawhney G, Singh B, Parsaik A, et al. Meta-analysis of clinical correlates of acute mortality in takotsubo cardiomyopathy. Am J Cardiol. 2014;113(8):1420-8.

3. Giza DE, Lopez-Mattei J, Vejpongsa P, Munoz E, lliescu G, Kitkungvan D, et al. Stress-Induced Cardiomyopathy in Cancer Patients. Am J Cardiol. 2017;120(12):2284-8.

4. Joy PS, Guddati AK, Shapira I. Outcomes of Takotsubo cardiomyopathy in hospitalized cancer patients. J Cancer Res Clin Oncol. 2018;144(8):1539-45.

5. Sattler K, El-Battrawy I, Lang S, Zhou X, Schramm K, Tülümen E, et al. Prevalence of cancer in Takotsubo cardiomyopathy: Short and long-term outcome. Int J Cardiol. 2017;238:159-65.

6. Templin C, Ghadri JR, Diekmann J, Napp LC, Bataiosu DR, Jaguszewski M, et al. Clinical Features and Outcomes of Takotsubo (Stress) Cardiomyopathy. N Engl J Med. 2015;373(10):929-38.

7. Desai A, Noor A, Joshi S, Kim AS. Takotsubo cardiomyopathy in cancer patients. Cardio-Oncol Lond Engl. 2019;5:7.

8. Citro R, Okura H, Ghadri JR, Izumi C, Meimoun P, Izumo M, et al. Multimodality imaging in takotsubo syndrome: a joint consensus document of the European Association of Cardiovascular Imaging (EACVI) and the Japanese Society of Echocardiography (JSE). Eur Heart J Cardiovasc Imaging. 2020;21(11):1184-1207.

9. Cheungpasitporn W, Kopecky SL, Specks U, Bharucha K, Fervenza FC. Non-ischemic cardiomyopathy after rituximab treatment for membranous nephropathy. J Ren Inj Prev. 2017;6(1):18-25.

10. Kanamori H, Tsutsumi Y, Mori A, Kawamura T, Obara S, Shimoyama N, et al. Delayed reduction in left ventricular function following treatment of non-Hodgkin's lymphoma with chemotherapy and rituximab, unrelated to acute infusion reaction. Cardiology. 2006;105(3):184-7.

11. Sharkey SW, Lesser JR, Zenovich AG, Maron MS, Lindberg J, Longe TF, et al. Acute and reversible cardiomyopathy provoked by stress in women from the United States. Circulation. 2005;111(4):472-9.

12. Halliday BP, Wassall R, Lota AS, Khalique Z, Gregson J, Newsome S, et al. Withdrawal of pharmacological treatment for heart failure in patients with recovered dilated cardiomyopathy (TRED-HF): an open-label, pilot, randomised trial. Lancet Lond Engl. 2019;393(10166):61-73.

\section{Figures}




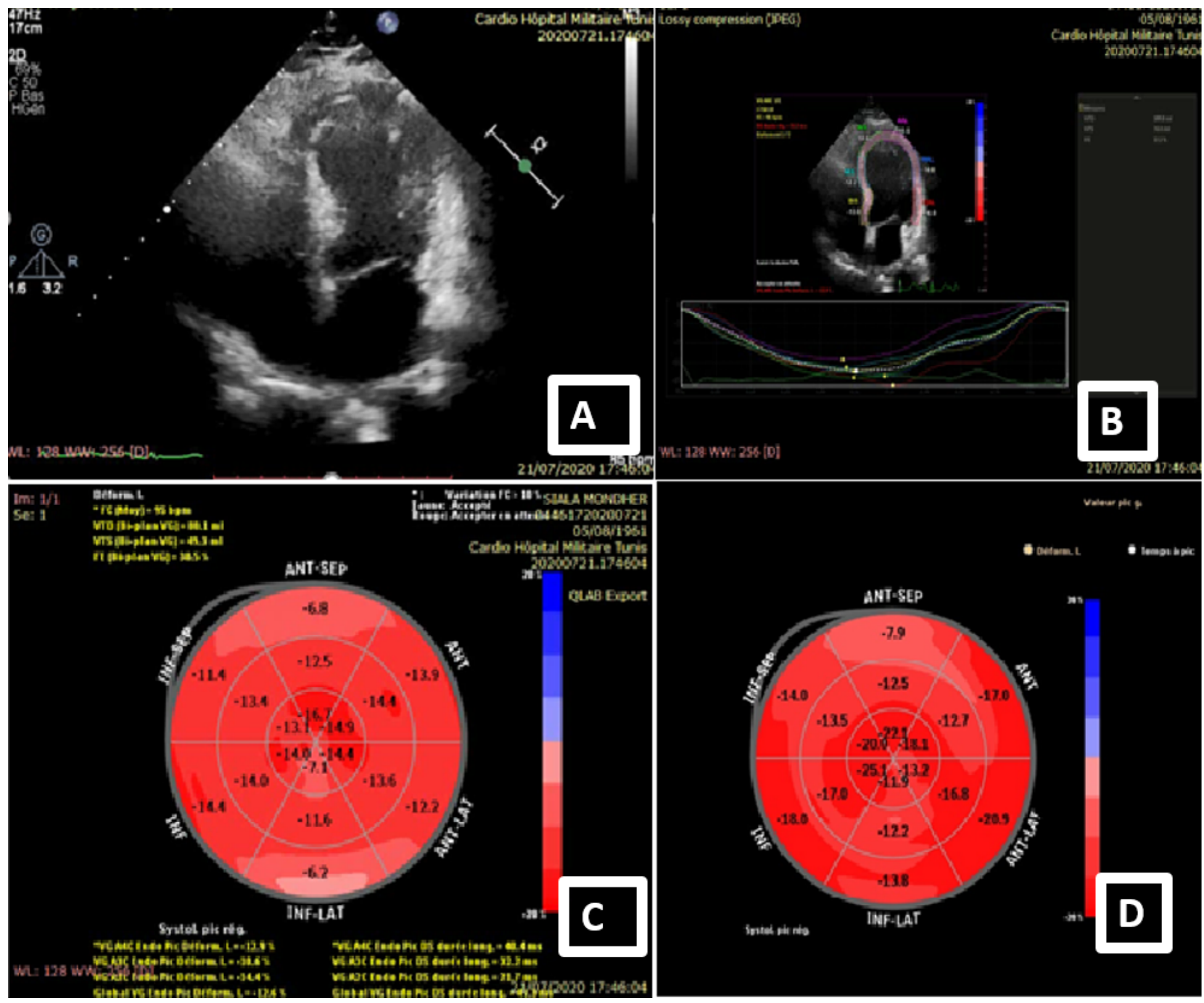

\section{Figure 1}

Two-dimensional transthoracic echocardiography, apical four- chambers view A: Apical ballooning during systole, B: Left ventricular Longitudinal 2D strain curve, C: 2D study and bul's eye, D: bul's eye after recovery of the left ventricular function. 




Figure 2

Coronary angiogram showing non obstructive lesion in right coronary artery 

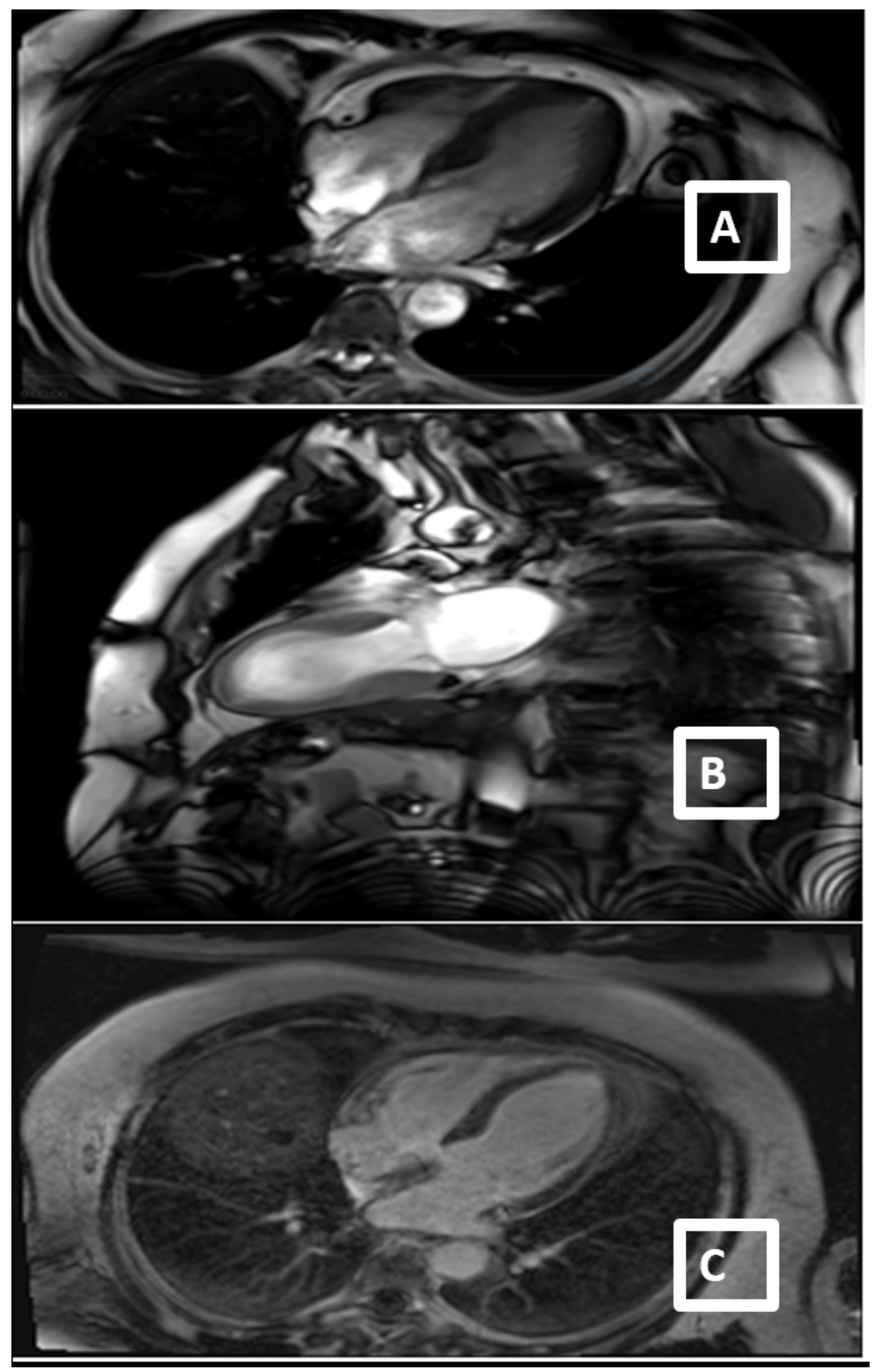

Figure 3

Cine cardiac magnetic resonance of ourpatient. A two- and four-chambers view of the left ventricle showed apical ballooning, transmural oedema. No ischemia, no signs of myocarditis, no myocardial infiltration. 